\title{
Desigualdades de género en trabajos de cuidados familiar y no remunerado: una revisión Iberoamericana.
}

Gender inequalities in family and unpaid care work: an Ibero-American review.

$$
\text { Constanza Gómez-Rubio*, Catalina Ganga-León }{ }^{* *} \text {, Wilson Rojas Paillalef*** }
$$

\section{Resumen}

Los cuidados se ven atravesados inexorablemente por el género, generando un problema de desigualdad y naturalización de éstos. Esta revisión sistemática indaga el conocimiento académico generado sobre el trabajo de cuidados no remunerado y familiar en Iberoamérica, dando cuenta de sus principales perspectivas y contenidos, y los factores de género asociados a las mismos. Consultamos cinco bases de datos, considerándose artículos empíricos publicados entre los años 2009 y 2014, con indexación ISI, SCOPUS y SCIELO, en idioma inglés, español y portugués. La muestra final fue de 51 artículos, sometidos a análisis de frecuencias y porcentajes, y análisis cualitativo de contenido. Los resultados evidencian la predominancia de la perspectiva biomédica al comprender los cuidados, y el escaso ejercicio reflexivo en torno a la comprensión que se posee de éste. Discutimos la necesidad de posicionar los cuidados como eje central de la vida humana, reorganizándolos y colectivizándolos socialmente.

Palabras claves: trabajo de cuidados no remunerado, cuidadora familiar, desigualdades de género, interdependencia, revisión.

\begin{abstract}
Care is inexorably crossed by gender, generating a problem of inequality and naturalization of these. This systematic review investigates the academic knowledge generated about unpaid and family care work in Ibero-America, showing its main perspectives and contents, and the gender factors associated with them. We consulted five databases, considering empirical articles published between 2009 and 2014, indexed ISI, SCOPUS and SCIELO, in English, Spanish and Portuguese. The final sample consisted of 51 articles, subjected to frequency and percentage analysis, and qualitative content analysis. The results show the predominance of the biomedical perspective in understanding care, and the lack of reflective exercise around the understanding of it. We discuss the need to position care as the central axis of human life, reorganizing and collecting socially.

\footnotetext{
* Mg. en Estudios de Género y Cultura, Psicóloga, Universidad de Chile. Becaria doctoral de la Comisión Nacional de Investigación Científica y Tecnológica, CONICYT y Universidad Autónoma de Barcelona. Este trabajo fue patrocinado por CONICYT PFCHA/ DOCTORADO EN EL EXTRANJERO 72160039. Correo electrónico: constanza.gomez@uab.cat

** Licenciada en Psicología, Universidad de Chile

*** Licenciado en Psicología, Universidad de Chile
} 
Revista Punto Género № 7. Mayo de 2017

ISSN 0719-0417 / 156 - 182

Key words: unpaid care work, family caregivers, gender inequalities, interdependence, review.

Fecha de recepción: 09 de octubre 2015

Fecha de aprobación: 13 de marzo 2017 


\section{Introducción}

Adentrarnos en los cuidados es transitar por un territorio complejo. Debemos al movimiento feminista europeo de la década del '70, el comienzo del debate sobre una antigua realidad que, por naturalizada, no había sido cuestionada: el conjunto de actividades necesarias para la supervivencia básica de las personas como campo específico de estudio, y cómo las mujeres a lo largo de la historia desarrollaron un complejo trabajo producto de cada cultura, muy alejado de la biología o la genética (Tobío, Agulló, Gómez, y Martín, 2010). Así, los cuidados se configuran como un aspecto fundamental e indispensable para la existencia, sostenibilidad y reproducción de la vida humana, pues todos/as necesitamos cuidados a lo largo de nuestras vidas. (Comas D’Argemir, 2014)

Siendo un tema de alta relevancia social, no existe una comprensión única del concepto, o muchas veces ni siquiera es especificado en las producciones académicas, existiendo una presuposición del término y su significado, es decir, asumiendo que todos/as compartiríamos una misma idea en torno al mismo. Sin embargo, es posible identificar ciertas perspectivas dominantes en su estudio, como por ejemplo, la noción de cuidado que la comprende desde un paradigma médico y económico. De este modo, es relevante hacer visibles estas presuposiciones, que operan a la base de prácticas sociales que perpetúan las desigualdades de género.

En esta línea, si hay algo característico en el trabajo de cuidados es que se trata de un ámbito siempre atravesado por el género, pues su distribución recae generalmente en las mujeres (Jelin, 2013; Batthyány, 2010; Arriagada y Todaro, 2012). Por ende, las diferentes producciones en torno a los cuidados se encuentran estrechamente vinculadas a las estructuras de poder y la desigualdad, operando ideológicamente en la reproducción del privilegio (Tronto, 1993). Así, Shahra Razavi (2007) comprende el cuidado como un trabajo dirigido no solo a personas con dependencia, sino también a los/as adultos/as autónomos/as, quienes serían generalmente hombres. Sira del Río (2004) los refiere como "dependientes sociales", vale decir, la inmensa mayoría de hombres que son dependientes porque no tienen ni la formación para cuidarse, ni el interés en hacerlo, lo cual acentúa la precariedad en el colectivo de las mujeres.

Por su parte, tal como expone María Jesús Izquierdo (2003), el cuidado suele ser atendido en situaciones de dependencia unidireccional y como una necesidad de carácter individual, frecuentemente adoptando una perspectiva física y material, en que "se concibe como ciudadano un individuo que asume tareas de provisión, protección y cura, el cual dispone de una infraestructura de apoyo para el cuidado de las personas dependientes y de sí mismo" (p. 3); dejando de lado la concepción de que el cuidado se da en relación a otros/as, vale decir, somos tanto cuidadores/as, como objeto de 
cuidados. En este sentido, los ideales de libertad, independencia y autosuficiencia que forman parte de las sociedades patriarcales y capitalistas, no son más que una fantasía que pretende hacernos olvidar que siempre necesitamos de los demás en algún grado y forma.

Al respecto, los cambios sociales, económicos, políticos y demográficos generados por la economía capitalista global de las últimas décadas, han perfilado la denominada "crisis de los cuidados", escenario que ha puesto en evidencia la "agudización de las dificultades de amplios sectores de la población para cuidarse, cuidar o ser cuidados" (Ezquerra, 2011: 176). Uno de los problemas fundamentales ligados a esta crisis, es la subordinación de las necesidades humanas a los mercados, en que las mujeres quedan en una posición desventajosa (Del Río, 2004). Por ende, debemos demandar una reorganización de las formas de reparto del trabajo de cuidados que requiere una sociedad, y reinterpretar "una necesidad típica del mundo privado como problema público y objeto de políticas" (Krmpotic y De leso, 2010: 96).

\section{Trabajo de cuidados familiar no remunerado en Iberoamérica: un problema de género}

En la mayor parte de los países iberoamericanos ${ }^{1}$ el bienestar cotidiano suele basarse, casi exclusivamente, en la institución familiar, no cuestionando el papel asistencialista del Estado respecto al cuidado (Castelló, 2009). En América Latina, pese a las distintas realidades de cada país, es posible reconocer que las políticas de mercado impulsadas desde los '90, han implicado un aumento en la carga de trabajo no remunerado de las mujeres (Martínez y Voorend, 2011). En este sentido, la disminución del gasto social por parte de los Estados afectó directamente las estrategias cotidianas de supervivencia de las familias y comunidades, acentuando la familiarización del cuidado, particularmente la intensificación del mismo por parte de las mujeres de clase media y clase baja, las que además de responsabilizarse del cuidado de su familia, lo hicieron también respecto a las familias de otras mujeres (Flores y Tena, 2014). En consecuencia, la demanda de cuidados en la región es atendida fundamentalmente por las familias y el mercado, generando diversos efectos en las mujeres, quienes son las principales responsables del cuidado de otros/as.

La fuerza simbólica del familismo, define una "cultura del cuidado" sustentada en convenciones patriarcales de género, que ven dicho trabajo como una obligación innata y natural de las mujeres (Letablier, 2007). De igual modo, en la región Latinoamericana, destaca la predominancia del maternalismo, idealizando la maternidad, a las mujeres en tanto madres, y el cuidado de los/as hijos/as (Flores y Tena, 2014). Este maternalismo exalta la diferencia entre hombres y mujeres en términos de sus capacidades, reforzando

\footnotetext{
${ }^{1}$ Entendemos Iberoamérica como aquél conjunto de países de América que fueron colonias de España y/o Portugal, así como los países colonizadores, otorgándole una raíz cultural, idiomática, entre otros, compartida en la región.
} 
la división sexual del trabajo y siendo, por tanto, un maternalismo patriarcal (Martínez y Voorend, 2009).

Así, el trabajo de cuidados no remunerado realizado en el ámbito familiar, se erige como el campo más amplio y encubierto del cuidado, donde las mujeres de las familias realizan cantidades enormes de trabajo invisibilizado (Aguirre, 2008). Se erige como una actividad obligatoria y desinteresada, y asimismo, se asume como parte natural de las expresiones familiares, particularmente como virtudes innatas femeninas, como el amor o la compasión (Letablier, 2007). Existen así, fuertes afinidades entre la comprensión del cuidado "altruista" y desinteresado, y los rasgos idealizados del género femenino, tal como plantea Simone de Beauvoir (1972): cuanto más cuida de las necesidades de los demás a expensas de las suyas, mejor madre, esposa o hija será a los ojos de los demás, e incluso de los propios. Desde el existencialismo, la autora sostiene que este tipo de cuidado implica, para quien lo brinda, des-responsabilizarse por sus propios intereses, actos, y la creación de una vida propia, sin hacerse cargo de la misma.

Debido a lo anterior, la dedicación de las mujeres al cuidado, tiene efectos significativos en sus alternativas laborales, ya que al ser socialmente las principales responsables del bienestar de otros/as, muchas veces deben enfrentar dificultades para conciliar familia y trabajo remunerado, o bien, deben excluirse de éste último (Batthyány, 2010). En Chile, por ejemplo, alrededor de 1.377 .352 mujeres se declararon inactivas producto de razones de cuidado de hijos/as y/o otras personas dependientes. A su vez, de la totalidad de personas que ejercen el cuidado de familiares, el $97,7 \%$ corresponde a mujeres, mientras que el 2,2\% a hombres. De igual modo, cabe señalar que de las mujeres que se encuentran inactivas por razones de cuidado familiar, la mayor parte cuenta con educación secundaria y primaria (Instituto Nacional de Estadísticas [INE], 2016a). Estas cifras resultan concordantes con la Encuesta Nacional de Uso del Tiempo realizada por el INE (2016b), en tanto en un día tipo, las mujeres destinan en promedio a nivel nacional 5,89 horas al trabajo no remunerado, mientras que los hombres destinan 2,74 horas. Estos datos permiten apreciar que la normatividad, la ideología de género y las prácticas en la división de tareas entre mujeres y hombres, limita las posibilidades de estos últimos para destinar tiempo al cuidado tanto de sus familias como de ellos mismos, contribuyendo a la inequidad social y de género (Arriagada y Todaro, 2012).

Para poder entender la complejidad de la problemática, debemos considerar el género en su interseccionalidad con otras categorías sociales -que pueden ser múltiples-, como el nivel socioeconómico (Wodak, 2015, entre otras), el que adquiere relevancia dado que implica la desigualdad entre aquellas mujeres que pueden acceder al mercado para adquirir servicios pagados de cuidados y aquellas que no cuentan con los recursos para hacerlo. En este sentido, la pobreza de ingresos se traduce en pobreza de tiempo, magnificando sus efectos (Jelin, 2013). Para Amartya Sen (2000), la pobreza debería ser comprendida no solo como la falta de ingresos, sino también como la privación de capacidades básicas. La relación entre el ingreso y las capacidades puede verse afectada 
por factores como la edad, el género, y las responsabilidades sociales vinculadas a éste, como por ejemplo la maternidad, ya que dichos factores pueden afectar y reducir la capacidad de una persona para percibir ingresos. De esta forma, el autor sostiene que la pobreza real puede ser mayor de lo que se vislumbra respecto al salario.

Dados estos aspectos, el presente artículo busca aproximarse al conocimiento académico generado durante los últimos años sobre el trabajo de cuidados no remunerado y familiar en Iberoamérica, dando cuenta de las principales características de los cuidados y factores de género asociados a las mismas. Para delimitar la búsqueda, se estableció como criterio de corte geográfico Iberoamérica, dado que es una región que comparte una historia y aspectos culturales en común.

La revisión correspondió a la literatura empírica publicada entre los años 2009 y 2014, con indexación ISI, SCOPUS y SCIELO, cuya elección estuvo dada por la importancia que éstas tienen en el discurso académico, siendo fuentes legitimadas en la búsqueda y publicación en la comunidad científica. Dicha revisión, posibilitó conocer qué tipo de estudios se realizan primordialmente, en relación a sus contenidos y enfoques, junto con identificar las incipientes tensiones y disrupciones en la investigación actual de este campo.

El aporte de este trabajo es principalmente teórico, dada la relevancia de evidenciar la necesidad de generar un mayor cuerpo investigativo sobre cuidados no remunerados en esta región, principalmente respecto a aquellos estudios publicados en las fuentes académicas ya mencionadas, y de carácter empírico. Adicionalmente, este tipo de revisiones permite explicitar la falta de perspectivas más críticas abordando la temática del cuidado indagado. En este sentido, a partir de lo revisado, establecemos propuestas de estudios para indagar formas alternativas y potenciales de cuidados, como la comunitaria, enfatizando en su relación con la noción de interdependencia propuesta desde el feminismo.

Para organizar el presente artículo, hemos dividido la información en tres apartados: primero, exponemos la metodología y el procedimiento de realización de la revisión sistemática, explicando los criterios de búsqueda, selección y análisis de la literatura. En el segundo, damos cuenta de los resultados obtenidos, primeramente brindando un panorama estadístico respecto a las publicaciones, y posteriormente en tres categorías temáticas cualitativas y comprensivas del conocimiento académico producido a la fecha. Finalmente, realizamos una discusión y nuestras conclusiones en torno a la problemática indagada.

\section{Metodología}

El presente artículo, lo realizamos en base a una revisión sistemática de estudios iberoamericanos orientados a la investigación del trabajo de cuidados no remunerado y 
familiar. Entendiendo por revisión sistemática "un estudio integrativo, observacional, retrospectivo, secundario". (Beltrán, 2005: 62)

El proceso de selección de artículos científicos siguió las indicaciones de la declaración Preferred Reporting Items for Systematic Reviews and Meta-Analyses (PRISMA) (Urrútia y Bonfill, 2010). De esta forma, consideramos artículos tanto cuantitativos como cualitativos, realizando el análisis de los mismos, en una primera instancia, a partir del cálculo estadístico de frecuencias y porcentajes, que dieron el contexto para efectuar un análisis de contenido desde un enfoque cualitativo. Cabe señalar, que este tipo de enfoque busca el establecimiento de relaciones y significados de un determinado tema en un contexto social, en lugar de la generalización de resultados a una población (Tarrés, 2004).

Realizamos la revisión entre octubre y diciembre del año 2014. Las bases de datos consultadas fueron: Web of Science, SCOPUS, SCIELO.org, PsycINFO, y Fuente Académica Premier. Los principales términos de búsqueda utilizados fueron: "trabajo de cuidados", "care work", "trabalho de cuidados", combinándolos con: "trabajo reproductivo", "trabajo doméstico", "trabajo no remunerado", "familia", "efectos cognitivos", "efectos afectivos" / "reproductive work", "house work", "unpaid work", "family", "cognitive effects", "affective effects" / "o trabalho reprodutivo", "trabalho doméstico", "trabalho não remunerado", "familia", "efeitos cognitivos", "efeitos emocionais". Cabe señalar, que no incluimos el término "cuidador informal" por una decisión política, pues consideramos que éste sigue una lógica mercantil que opera como pauta hegemónica aplicable a las tareas del cuidado no remunerado. Así, el calificativo informal juega en contra de una actividad realizada principalmente por mujeres hacia el cuidado familiar en condiciones de estricta formalidad y disponibilidad en su cotidianeidad. (Torns, 2008)

Seleccionamos estudios empíricos originales publicados en revistas científicas con revisión por pares, que cumplieran con los siguientes criterios de inclusión: a) abordaran la problemática de trabajo de cuidado familiar no remunerado, b) fueran de carácter empírico y con datos primarios provenientes de países de lberoamérica, c) se encontraran en revistas con indexación ISI, SCOPUS y/o SCIELO, para asegurar su calidad, d) fuesen publicados entre los años 2009 y 2014, en idioma español, inglés o portugués. El corte temporal de cinco años responde al criterio de validez utilizado por la comunidad científica. (Urrútia y Bonfill, 2010)

Excluimos los siguientes tipos de artículos: revisiones sistemáticas, meta-análisis y teóricos, editoriales de revistas, artículos periodísticos, artículos que abordaran el trabajo de cuidado desde lo remunerado o voluntariados, artículos en idiomas diferentes al español, inglés y portugués, y artículos que utilizaran datos secundarios.

\section{Procedimiento}


La búsqueda de artículos arrojó 1.023.806 de resultados en las bases de datos. Estos fueron brutos y totales en relación a los términos de búsqueda mencionados anteriormente, sin aplicar los criterios de inclusión y exclusión.

De la totalidad de los datos arrojados, 90 cumplían con los criterios de inclusión. De éstos, 39 se encontraban duplicados por lo que fueron eliminados, quedando un total de 51 artículos a incluir en el análisis. Cabe destacar que, pese a la gran cantidad de resultados, muchos referían al trabajo de cuidado remunerado, principalmente desde el trabajo de enfermería. Así también, muchos de los debates sobre el concepto de cuidado y perspectivas feministas eran abordados en literatura de carácter teórico -lo que se refleja en que sólo 14 de los 51 documentos empíricos incluidos referían explícitamente a su comprensión del cuidado, aunque gran parte de ellos lo hacían en referencia a la figura del "cuidador informal"-, o los estudios se encontraban en idiomas diferentes a los estipulados, por lo que fueron excluidos.

En el proceso de revisión, creamos una base de datos con 18 códigos de análisis iniciales de información, para la organización del material, tal como se observa en la Tabla 1.

Tabla 1. Definición de códigos de análisis iniciales

\begin{tabular}{|c|c|}
\hline Nombre de la categoría & Definición \\
\hline Autor/a & Nombre de autores/as y coautores/as \\
\hline Año & Fecha de la publicación \\
\hline Revista & Revista de origen de la publicación del artículo \\
\hline Temática de la revista & Disciplina de la revista catalogada en las bases de datos \\
\hline País & País de origen muestral \\
\hline Objetivos & El objetivo u objetivos del artículo \\
\hline Metodología & Estudio cualitativo, cuantitativo o mixto \\
\hline Características muestrales & Caracterización de los/as participantes en los estudios \\
\hline $\begin{array}{l}\text { Técnicas de producción de } \\
\text { información }\end{array}$ & $\begin{array}{l}\text { Herramientas o instrumentos utilizados para la producción } \\
\text { o recolección de información }\end{array}$ \\
\hline Análisis de información & Técnicas de análisis de la información producida o recogida \\
\hline Definición del cuidado & Concepción explícita de los cuidados y trabajo de cuidados \\
\hline Abordaje de los cuidados & Concepción implícita de los cuidados \\
\hline Quién realiza el cuidado & $\begin{array}{l}\text { Mención explícita de las personas que ejercen el trabajo de } \\
\text { cuidado }\end{array}$ \\
\hline $\begin{array}{l}\text { A quien se dirige el } \\
\text { cuidado } \\
\text { Motivos por los que se } \\
\text { cuida }\end{array}$ & $\begin{array}{l}\text { Mención explícita de las personas a quienes se cuida } \\
\text { Razones explícitas e implícitas por las que se ejerce el } \\
\text { trabajo de cuidados } \\
\text { Conductas que son comprendidas como cuidado } \\
\text { Efectos físicos, psicológicos y sociales. }\end{array}$ \\
\hline $\begin{array}{l}\text { Tipo de cuidados } \\
\text { Efectos del cuidado en } \\
\text { quien cuida }\end{array}$ & \\
\hline
\end{tabular}




\section{Elaboración propia.}

Los códigos fueron creados de forma mixta, vale decir, hubo algunos que se establecieron con anterioridad al análisis de los documentos, en tanto otros surgieron como parte del análisis de los datos.

Inicialmente, organizamos el material según las frecuencias de ciertos códigos. Para esto, creamos tablas en el programa Microsoft Excel asociadas a la información de los artículos publicados, así como de las fuentes de publicación. De este modo, el análisis se realizó en torno a tres ejes: a) publicaciones y revistas; b) contenido de las publicaciones; y c) aspectos metodológicos de los artículos seleccionados. Posteriormente, calculamos los porcentajes y generamos tablas y gráficos respecto a la información recopilada.

Una vez realizado el procedimiento anterior, trabajamos en el análisis de contenido temático de los artículos seleccionados, que nos permitió estudiar aquello explícito e implícito de éstos, así como también profundizar en su interpretación y contexto de producción. (Andreú, 2002)

De esta forma, los artículos fueron leídos y agrupados por afinidad temática con ayuda de la organización realizada en el proceso de revisión [ver Tabla 1]. Luego, los códigos iniciales de análisis de información se asociaron en tres categorías: a) Comprensión de quien cuida, b) Comprensión del cuidado, y c) Roles sociales para hombres y mujeres. Estas categorías son desarrolladas en el apartado de resultados.

Por último, queremos destacar que nuestra intención no es plantear que no existan otras fuentes de conocimiento válidas, sino que la revisión se centró en las indexaciones que académicamente son legitimadas como fuentes importantes para la divulgación de investigaciones. Sabemos que el conocimiento sobre la problemática aquí abordada no se agota, de manera alguna, en los artículos revisados, y que existen más cuerpos de conocimiento en otros medios y de otros tipos, así como también pueden existir investigaciones en otras lenguas y zonas geográficas. Por esto, lo presentado aquí se circunscribe a los criterios de inclusión utilizados y al procedimiento descrito.

\section{Resultados}

De los artículos trabajados, constatamos que los estudios empíricos sobre cuidados no remunerados en Iberoamérica, publicados en las bases de datos ya mencionadas, van adquiriendo relevancia, lo que se refleja en la constante tendencia a generar publicaciones durante el período considerado (2009-2014). Tal como es posible apreciar en el Gráfico 1, el año 2012 es el que concentra un mayor número de investigaciones publicadas (27,5\%), seguido por los años 2013 (21,6\%), 2011 (15,7\%), 2014 (13,7\%), 2009 $(11,8 \%)$, y $2010(9,8 \%)$. 
Gráfico 1: Publicaciones por año.

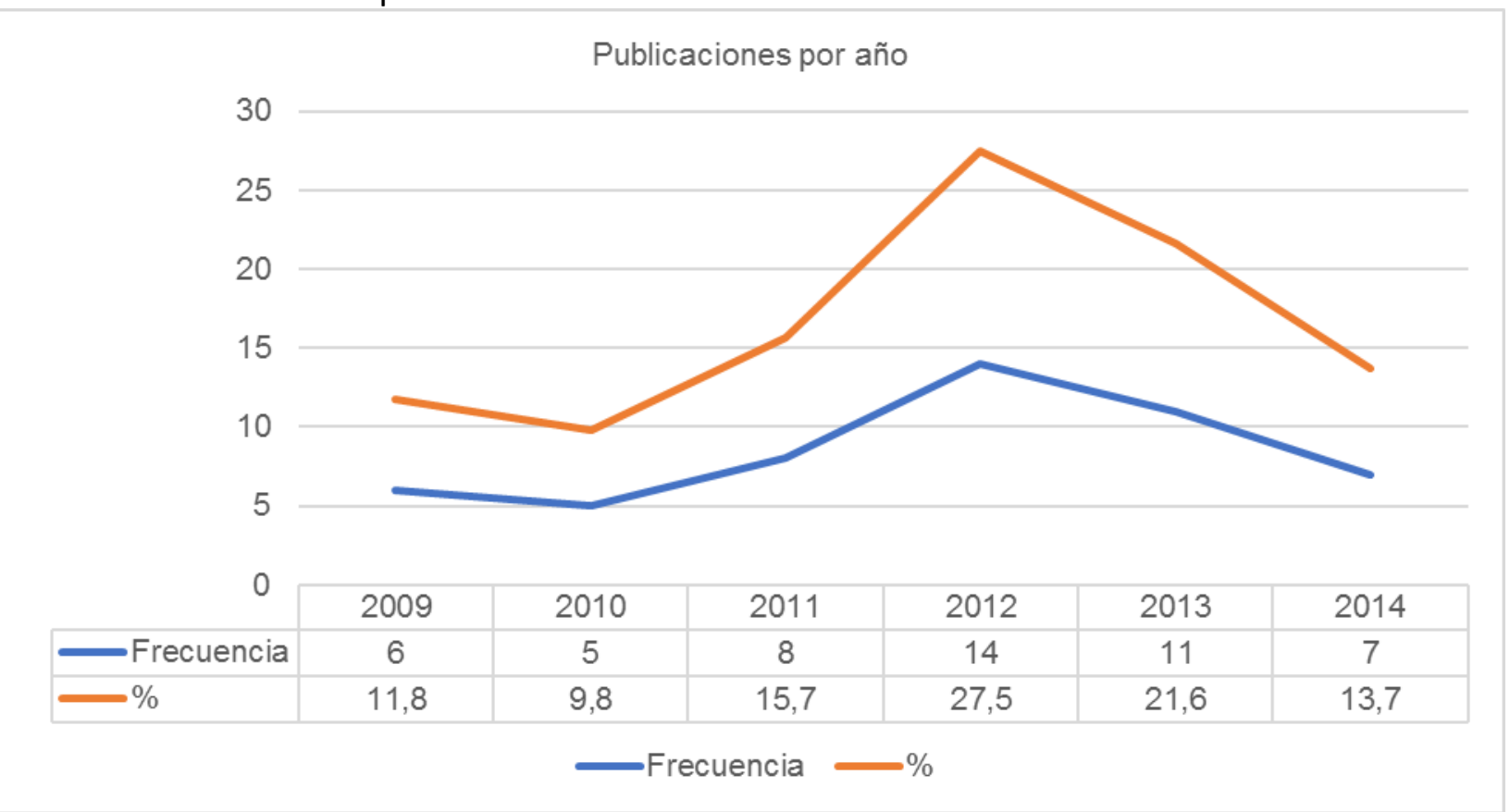

Fuente: Elaboración propia.

Asimismo, el $49 \%$ de los estudios corresponden a publicaciones con muestras en Brasil, y un 23,5\% a publicaciones en España, seguido muy por debajo por Colombia, Portugal y Chile con 5,9\% cada uno. Mientras que, entre los países con menos publicaciones, se cuentan: Cuba, México, Argentina, Perú y otros países de la región con un $2 \%$, lo que equivale a una publicación respectivamente (Tabla 2).

Tabla 2: Procedencia de los artículos

\begin{tabular}{lll}
\hline \multicolumn{2}{l}{ Procedencia de los artículos } \\
\hline País de la muestra & Frecuencia & $\%$ \\
\hline Argentina & 1 & 2,0 \\
\hline Brasil & 25 & 49,0 \\
\hline Chile & 2 & 3,9 \\
\hline Colombia & 3 & 5,9 \\
\hline Cuba & 1 & 2,0 \\
\hline
\end{tabular}




\begin{tabular}{lll} 
México & 1 & 2,0 \\
\hline Perú & 1 & 2,0 \\
\hline Portugal & 3 & 5,9 \\
\hline España & 12 & 23,5 \\
\hline Otros & 2 & 3,9 \\
\hline Total & 51 & 100,0 \\
\hline
\end{tabular}

Elaboración propia.

Respecto a las fuentes de publicación, fueron 39 revistas, entre las que se destaca "Texto e Contexto Enfermagem" con un 9,8\% de las publicaciones, seguida por "Aquichan" con 5,9\%. Por debajo y de manera homogénea siguen siete revistas con un $3,9 \%$ y veintinueve con $2 \%$, tal como se aprecia en la Tabla 3.

Tabla 3: Distribución de publicaciones por revista

\begin{tabular}{lll}
\hline Distribución de publicaciones por revista & & \\
\hline Revistas con más publicaciones & Frecuencia & $\%$ \\
\hline Texto e Contexto Enfermagem & 5 & 9,8 \\
\hline Aquichan & 3 & 5,9 \\
\hline Revista Gaúcha Enfermagem & 2 & 3,9 \\
\hline Escola Anna Nery Revista Enfermagem & 2 & 3,9 \\
\hline Revista Brasileira de Geriatria e Gerontologia & 2 & 3,9 \\
\hline Revista Latino-Americana de Enfermagem & 2 & 3,9 \\
\hline Revista Latinoamericana de Ciencias Sociales, & 2 & 3,9 \\
Niñez y Juventud & 2 & 3,9 \\
\hline Enfermería Global & 2 & 3,9 \\
\hline Index de Enfermería & 1 & 2,0 \\
\hline Midwifery & 1 & 2,0 \\
\hline Clínica y Salud & 1 & 2,0 \\
\hline Enfermería Clínica & 1 & \\
\hline Infancia y Aprendizaje: Journal for the Study & of & 2,0 \\
\hline Education and Development & 1 & 2,0 \\
\hline Interamerican Journal of Psychology & 1 & 2,0 \\
\hline Matronas Profesion & 1 & 2,0 \\
\hline Medicina Paliativa & 1 & 2,0 \\
\hline Qualitative Health Research & 1 & 2,0 \\
\hline Revista Facultad Nacional de Salud Pública & 1 & 2,0 \\
\hline Suma Psicológica & 1 & 2,0 \\
\hline Sociologia, Problemas e Praticas & 1 & 2,0 \\
\hline Revista de Enfermagem Referência & 1 & 2,0 \\
\hline ACTA Paulista de Enfermagem & & \\
\hline
\end{tabular}


American Journal of Hospice and Palliative

Medicine 1

Archivos Argentinos de Pediatría 1

Ciencia e Saude Coletiva 1

Ciencia y Enfermería 1

MEDISAN 1

Pensamiento Psicológico 1

Psicologia \& Sociedade 1

Psicologia: Teoria e Pesquisa 1

Revista da Escola de Enfermagem da USP

Revista de Salud Pública 1

Revista Brasileira de Enfermagem REBEn 1

Revista Brasileira de Psiquiatria 1

Revista Colombiana de Psicología 1

Revista Enfermagem 1

Temas em Psicologia

Terapia Psicológica

Total 12,0

12,0

12,0

12,0

12,0

12,0

12,0

$1 \quad 2,0$

12,0

12,0

12,0

$1 \quad 2,0$

12,0

12,0

12,0

12,0

$51 \quad 100,0$

Elaboración propia

La distribución disciplinar de las revistas responden mayoritariamente a aquellas de Enfermería, Medicina y Ciencias de la Salud, lo que se refleja en un $68,42 \%$ de los artículos publicados, mientras que aquellos relacionados con Psicología, Ciencias Sociales y Humanas corresponden a un 31,58\%. Así, tal como se observa en el Gráfico 2, las revistas de enfermería son las que cuentan con más estudios publicados, al igual que las de medicina $(23,68 \%)$.

Gráfico 2: Distribución disciplinar de revistas 


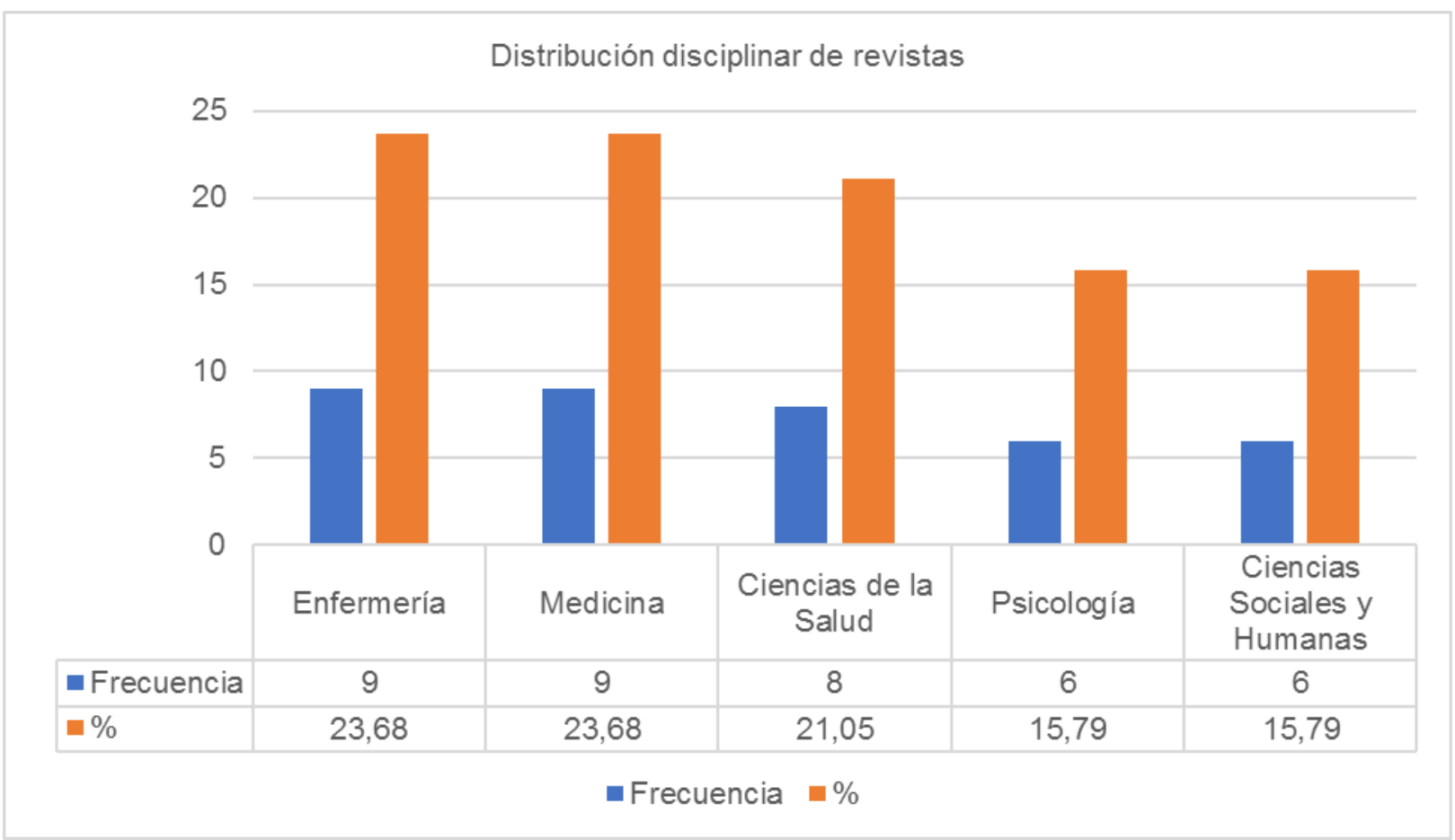

Fuente: Elaboración propia

En relación a la metodología utilizada en las investigaciones, el 56,9\% fueron de carácter cualitativa, el $41,2 \%$ cuantitativa y el $2 \%$ mixtas, es decir, contaban con metodologías cualitativas y cuantitativas.

Por su parte, la mayor parte de los/as participantes fueron hombres y mujeres en una misma muestra (39,2\%), seguido por muestras exclusivas de mujeres $(23,5 \%)$ y otras formas de organización muestral (15,7\%). Posteriormente, y de manera bastante inferior, aparecen muestras exclusivas de hombres $(5,9 \%)$, de parejas $(3,9 \%)$, y diadas entre cuidadora/persona cuidada (3,9\%). El $7,8 \%$ de las publicaciones no especificaron mayormente a los/as participantes.

En relación a la identificación muestral realizada, los artículos señalaban a las mujeres, sin mayor descripción ni caracterización, como la población que comúnmente ejerce trabajo de cuidados, con un 47,1\%; seguido muy por debajo por mujeres con hijos/as y mujeres con baja escolaridad, con 3,9\% cada una. Además, un $2 \%$ identificó a las mujeres en situación de pobreza, es decir, solamente un artículo. Igualmente, un 43,1\% no contenía esta información.

Las temáticas abordadas por la literatura académica se enfocaban, principalmente, en el estudio de personas enfermas (33,3\%), seguido de la maternidad/paternidad $(29,4 \%)$. Un número inferior de estudios lo ha hecho en personas mayores $(21,6 \%)$, y otros con distintas poblaciones que no alcanzan a ser significativas individualmente $(15,7 \%)$. 
Asimismo, la mayor parte de las investigaciones no indagaba en los motivos por los que se ejerce el trabajo de cuidado (70,6\%), y en aquellas que sí lo exploraban, el principal motivo correspondía a la obligación $(11,8 \%)$, seguido del mandato de género o norma social (7,8\%); luego la naturaleza y la voluntad (3,9\%), y en último lugar, por amor o afecto, con un $2 \%$.

Por otro lado, solo 21 artículos explicitaban las principales conductas que reflejaban cuidados: la administración y preparación de los alimentos, junto con la dieta, fue la conducta más destacada (28,6\%), luego, la administración de medicamentos $(23,8 \%)$, seguido por el transporte y la asistencia a citas médicas (19\%), finalizando con jugar y/o criar, y la mantención de la higiene y vestimenta, con 14,3\% respectivamente.

Finalmente, basándonos en el contenido de los 51 estudios seleccionados, construimos tres categorías con las cuales es posible englobar las investigaciones sobre trabajo de cuidados en Iberoamérica: a) Comprensión de quien cuida, b) Comprensión del cuidado, y c) Roles sociales para hombres y mujeres; que desarrollamos a continuación.

\section{A. Comprensión de quien cuida}

La mayor parte de las personas que realizan labores de cuidado son mujeres (Carrion et al., 2013), lo que tiene relación con que una cantidad considerable de investigaciones estuvo compuesta por una muestra exclusivamente de éstas, en tanto una cantidad bastante inferior, lo hizo solo de hombres. Cabe destacar que la mera inclusión de mujeres, no implica necesariamente que los estudios hayan tenido un enfoque de género o una perspectiva feminista. Esto se refleja en el uso acrítico del lenguaje, por medio del término genérico "cuidador", asociado a una masculinización o neutralización del concepto -señalado en estudios como el de Magaña et al. (2011)-, en lugar de "cuidadora", cuyo uso contribuiría a visibilizar y reivindicar el trabajo femenino, y que queda oculto por medio de esta designación.

Por otra parte, gran parte de los estudios, distinguen entre cuidadoras/es formales e informales. Las/os primeras/os son entendidas/os como aquellas personas que trabajan remuneradamente en la labor de cuidado; mientras las/os segundas/os, serían quienes no realizan esta labor de modo remunerado, en general familiares o personas cercanas a la persona cuidada. Podemos apreciar el uso recurrente del binarismo "formal/informal", referido a las labores de mercado, en tanto lo formal se establece como aquello que opera dentro del mismo, mientras lo segundo, operaría por fuera, asociándose a valoraciones diferenciales entre una y otra. Asimismo, este binarismo se asocia con lo "público/privado", donde lo formal se relacionaría con lo público, que es validado socialmente; mientras lo informal se asocia a lo privado, más devaluado e invisibilizado. En este sentido, el uso recurrente en las investigaciones del término "informal", tendría como efecto evidenciar una comprensión del cuidado desde un punto de vista mercantilista. 
En esta línea, los tipos de familiares cuidadoras/es predominantes en los estudios, fueron madre, esposa, hija/o, abuela, hermana/o o nuera (Sousa et al., 2011; Villarejo et al., 2012). Los sujetos cuidados suelen ser personas con algún grado de dependencia (adultos/as mayores, niños/as, recién nacidos/as y adolescentes). A su vez, gran cantidad de ellas, poseen enfermedades discapacitantes (físicas, cognitivas, mentales y/o congénitas).

Respecto de las/os cuidadoras/es, la mayoría informó estar casada/o o tener una pareja, y poseer bajos niveles educacionales. La mayor parte no tenía un trabajo remunerado, ya que, en general, las exigencias de cuidado las/os llevó a trabajar menos horas o abandonar su empleo, aumentando la pobreza y vulnerabilidad familiar, quedando imposibilitadas/os de delegar el trabajo de cuidado al mercado, es decir, remunerar a una tercera persona para estas labores (e.g. Carrion et al., 2013; Casado-Mejía et al., 2013; De São José, 2012; Magaña et al., 2011; Stackfleth et al., 2012, entre otros).

Por otro lado, las/os cuidadoras/es presentaron problemas emocionales como ansiedad, estrés y depresión, derivando en un uso permanente de fármacos. Esto se presenta también a modo de angustia, trastornos del sueño, cambios emocionales, desesperanza y sentimientos de culpa (e.g. Aldana-González et al., 2011; Larrañaga et al., 2009; Truzzi et al., 2012, entre otros). Lo anterior puede asociarse a las dificultades técnicas y de conocimiento que presentan las personas que cuidan, que suelen concebirse como ineptitud y cuestionamiento a las propias capacidades, traduciéndose en sentimientos negativos por parte de ésta/e frente a la situación del cuidado (e.g. De Souza et al., 2010; Guerini et al., 2012; Weisbrot et al., 2012, entre otros).

Un aspecto poco explorado por las investigaciones, son las repercusiones en las dinámicas y estructuras familiares. Este factor se integra con el costo social que sufre la/el cuidador/a, el que se refleja en la postergación de su propia vida, cuando no existen otras alternativas para el cuidado (Aldana-González et., al 2011; Casado-Mejía et al., 2013). De esta forma, Lizyana Vieira et al. (2012) concluyen que la vida de la/el cuidador/a se configura y adapta en función de la persona cuidada, dejando de lado los intereses propios, esperando que la actividad finalice para volver a tener una vida "normal".

Adicionalmente, los estudios reflejan la falta de apoyo como una de las principales dificultades para las personas que cuidan, destacando la escasez de apoyo familiar, apoyo institucional, y red de apoyo comunitaria (e.g. Carrion et al., 2013; Casado-Mejía et al., 2013; Dos Santos et al., 2012, entre otros). Asimismo, las políticas públicas aparecen como un aporte, pero insuficiente para las/os cuidadoras/es. En este sentido, resultan de interés las conclusiones de María Del Río-Lozano et al. (2013) y Rosa Casado-Mejía et al.(2013), que plantean que aunque el hecho de cuidar a otra persona es algo desgastante tanto para mujeres como para hombres, estos últimos buscan sobrellevar el cuidado solicitando ayudas institucionales, ayuda de otras personas o contratando a una persona 
que ejerza el cuidado para salvaguardar un espacio personal; en contraste con las mujeres, que no suelen utilizar las redes de apoyo institucionales disponibles. En esta línea, otro estudio sostiene que cuando una madre recibe apoyo de un/a tercera/o en el cuidado de sus hijos/as, esto le genera sentimientos de culpa y cuestionamiento sobre sus capacidades de cuidado (Moreno-Zavaleta et al., 2014).

Lo anterior podría vincularse con los porcentajes respecto a los motivos por lo que se ejerce el cuidado, los que responden principalmente a una obligación y a un mandato de género, siendo esperable que las mujeres realicen los cuidados. En este sentido, a través del cruce de nuestro análisis, se podría en parte explicar que para ellas sería difícil escapar del mandato social de género, mientras para ellos, el cuidado no es un mandato central en su rol social, por lo que sería esperable de éstos que busquen ayuda en otras redes.

Referido en menor medida por los estudios, se encuentran los sentimientos positivos existentes en la labor de cuidado, donde se vuelve clave la aceptación de las condiciones en las que se encuentra la persona cuidada, así como también el experimentar la situación de cuidado como una instancia de crecimiento personal. También, las muestras de cariño hacia quien se cuida y el bienestar de éste/a, generan gratificación y satisfacción por parte de la/el cuidador/a (Bolla et al., 2013; Fratezi et al., 2011; Henn et al., 2010; Weisbrot et al., 2012). Esto concuerda con el análisis estadístico respecto a los motivos por lo que se cuida, en que el afecto como motivo del cuidado es el que menos se esgrime, y por lo tanto, pudiese inferirse que la satisfacción asociada al mismo sería poco evidenciado, y/o se invisibiliza éste en relación a otros motivos en los artículos estudiados.

\section{B. Comprensión del cuidado}

En general, fue menor la cantidad de estudios que brindaban una comprensión respecto al cuidado. Principalmente, puede caracterizarse como una conjunción de tareas, tales como la alimentación, administración de medicamentos, transporte o higiene de la persona cuidada, cuya finalidad es la preservación de la integridad física (e.g. Bustamante et al., 2011; De São José, 2012; De Sousa et al., 2009, entre otros). En menor medida, estudios como el de Irene Magaña et al. (2011), también incluyeron actividades de crianza y educación de hijos/as, el cuidado de los miembros que trabajan remuneradamente, así como el mantenimiento del hogar.

A su vez, respecto del cuidado de enfermos/as, tercera edad o discapacitados/as, algunas de las labores de cuidado son acompañarles a instituciones de salud, realizar actividades de asistencia médica (como vendajes, curaciones, cuidado de aparatos médicos y/o administración de medicamentos), y realizar actividades permanentes en función de mantener y controlar a enfermos/as crónicos/as, o a personas con condiciones especiales 
de capacidad física y/o mental (Magaña et al., 2011; Silva et al., 2013). El cuidado se comprende como una actitud constante que incluye esfuerzo y dedicación, así como entrega, atención y cariño hacia otro/a (Bedoya-Hernández, 2013; Dos Anjos et al., 2014; Gálvez et al., 2013; Henn et al., 2010; Silva et al., 2013). Desde este enfoque, desprendemos que frecuentemente en los estudios, el cuidado se vincula a la enfermedad y a lo patológico, restringiendo aquellas perspectivas desde las cuales se puede entender el cuidado -las que además no suelen ser explicitadas-. Esto contribuye a la reproducción de una noción hegemónica sobre los cuidados, que los entiende desde un paradigma biomédico, dificultando la conformación de debates sobre el cuidado y su comprensión. Esto podría tener efectos en las prácticas sociales cotidianas de las personas, reproduciéndose la desigualdad de género, al entenderse el cuidado de manera unidireccional y en estados humanos de "excepcionalidad", invisibilizando que el cuidado es una responsabilidad pública y de todos/as, y no responde únicamente a estados o periodos específicos de la vida, sino que perduran a lo largo de ésta.

Un aspecto importante para comprender el cuidado, es ver de qué forma éste es asumido por quien cuida. Por un lado, la evidencia indica que muchas veces el cuidado se designa e impone hacia una persona por parte de la familia, sustentado en un deber y una obligación moral (e.g. Dos Santos et al., 2010; Fratezi et al., 2011; Guerini et al., 2012, entre otros). Otros estudios refieren que la transición a convertirse en cuidador/a de un familiar, es concebida como parte del ciclo de vida, exhibiéndose un fuerte componente afectivo en la adopción de este rol (Del Río-Lozano et al., 2013). El estudio de Isabel Larrañaga et al. (2009) refiere a lo anterior como "solidaridad intergeneracional", la cual es entendida, principalmente por cuidadoras mayores, como la obligación familiar de cuidar, naturalizándose en un valor social y una función propia de las mujeres.

No obstante lo anterior, investigaciones como las de Mauricio Bedoya-Hernández (2013), Vania Bustamante et al. (2011), y María Moreno-Zavaleta et al. (2014), han marcado la diferencia en esta revisión, visibilizando otros tipos de cuidado. Estos tres estudios comparten su interés por indagar el cuidado y crianza de niños/as en comunidades en contextos de pobreza de Latinoamérica.

Bedoya-Hernández (2013) estudia hogares comunitarios de Antioquía, donde se presentan figuras como las "madres comunitarias", quienes cuidan y son cuidadas por niños/as, las familias de éstos/as, otras madres comunitarias y por sus propias familias. En este sentido, el estudio se enfoca en comprender el cuidado como una red de actores sociales involucrados/as, y no como una responsabilidad propia de un sujeto en particular, superando el estigma de la madre como única responsable del cuidado (Moreno-Zavaleta et al., 2014).

C. Roles sociales para hombres y mujeres 
Suele plantearse que la maternidad es el rol principal de las mujeres (Barona-Vilar et al., 2013). Los argumentos que legitiman esta tesis son de carácter social-biologicistas o esencialistas asociados, por ejemplo, al instinto maternal y a la capacidad natural para ejercer cuidado de personas (Rodríguez et al., 2014). Esto repercute en la decisión familiar de quién es la persona más idónea para cuidar, lo que se traduce en una fuerte presión social porque dicha tarea la cumpla una mujer en la familia (Del Río-Lozano et al., 2013). Esto es concordante con la obligación de cuidado por mandato de género analizado anteriormente.

El estudio de Mara Da Silva et al. (2012), da cuenta que el ejercicio del rol de mujer cuidadora se extiende a otras funciones sociales, por ejemplo, el cuidado de niños/as se asocia a la función de "educadora", el cuidado de personas con afecciones de salud se asocia a la función de "enfermera" y, finalmente, las tareas de higiene y relacionadas con el mantenimiento del hogar se asocian a las "tareas domésticas". De esta forma, se desprende que en el mundo laboral y profesional surgen habilidades socialmente atribuidas a lo femenino, tales como las relacionadas con el cuidado, reproduciendo la llamada "maternidad simbólica". Es importante no perder de vista que esta reproducción no responde puramente a una elección de carácter individual, sino implica una relación dialéctica entre las decisiones personales y las condiciones socio-estructurales.

Por otra parte, al abordar el rol del hombre en relación al cuidado de personas, rara vez aflora el sentimiento de deber moral de cuidar, por tanto, no se valora negativamente el no asumir este tipo de labores (Larrañaga et al., 2009). No obstante, cuando son asumidas se evidencia una valoración altamente positiva, calificando a estos hombres de "héroes" o "gente especial" (Del Río-Lozano et al., 2013).

El estudio de Carmen Barona-Vilar et al. (2013), que explora las experiencias y percepciones de paternidad y maternidad en cuidado de salud en mujeres latinoamericanas residentes en España, da cuenta que las experiencias subjetivas sobre estos aspectos son influidas por la cultura del país de origen, respecto de las asignaciones y valoraciones de género, evidenciándose un modelo tradicional en el patrón de relación entre hombres y mujeres, donde éstas últimas consideran la maternidad como el rol principal en la experiencia femenina.

Finalmente, al indagar en el rol del padre, se menciona que por tradición, éstos son los encargados de proveer a la familia, aun cuando éste asuma otras responsabilidades en el hogar (Fernandes de Oliveira et al., 2009; Henn et al., 2010). En cambio, respecto del rol de la madre, se plantea que la responsabilidad del cuidado y la protección de los/as hijos/as son características inherentes a éste (Carrion et al., 2013; Sanches, 2012; Wottrich et al., 2014). Este aspecto se relaciona con la división sexual del trabajo, es decir, con aquella especialización de actividades y labores atribuidas por sexo, conllevando una valoración social diferenciada, que se traduce en un trabajo remunerado y valorado, generalmente masculino, y otro no remunerado, principalmente femenino. 
Consecuentemente, esto plantea un modelo de familia heteropatriarcal conformada por padre, madre e hijos/as, reproduciendo los roles tradicionales de género e invisibilizando la diversidad de familias existentes.

\section{Discusión y Conclusiones}

En el presente artículo nos aproximamos al conocimiento académico generado entre los años 2009 al 2014, sobre el trabajo de cuidados familiar y no remunerado en Iberoamérica. Esto nos permitió visibilizar que la mayor parte de la literatura académica y empírica basada en los criterios de inclusión especificados previamente, no otorga una comprensión acabada y explícita respecto al cuidado, lo cual exhibiría una presuposición de su significado, asociado al mantenimiento físico de sujetos, en su mayoría, dependientes, en que el aspecto físico y corporal, otorga signos más visibles de la necesidad de cuidado. Esto contribuye a la consolidación de una normatividad corporal, generando un binarismo que segrega aquellos cuerpos "anormales" o necesitados de cuidados, asociándolos a la dependencia; mientras se vinculan los cuerpos "normales" a la autonomía e independencia absoluta, lo cual concuerda con lo señalado por Izquierdo (2003), en tanto se trata de una falacia que conforma nuestras sociedades patriarcales y capitalistas, que opaca el hecho de que siempre necesitamos de los demás.

En esta línea, mayoritariamente los estudios abordan el cuidado como una actividad individual, tradicionalmente femenina, sin realizar grandes cuestionamientos 0 reflexiones respecto a dicha concepción. Esto puede ligarse a que el conocimiento académico en su mayoría proviene de disciplinas vinculadas a las ciencias de la salud y la medicina, existiendo presuposiciones del orden biologicista, patologizante y esencialista respecto al cuidado, que no era problematizado. Por su parte, algunos pocos estudios con un enfoque desde las ciencias sociales, daban cuenta con una problematización algo mayor de las desigualdades de género en el cuidado, traducida habitualmente, en la naturalización de las habilidades femeninas para esta labor, conllevando a una devaluación de la contribución que éstas realizan al bienestar cotidiano y la reproducción de la vida humana. En este sentido, resulta relevante evidenciar que, pese a la centralidad de los cuidados en la sostenibilidad de la vida -como es señalado en parte de la literatura con enfoques feministas y/o críticos, tales como los trabajos de Dolors Comas D'Argemir (2014) o Sira del Río (2004), por mencionar algunas-, el abordaje de los estudios trabajados no se condice e incluso omite dicha centralidad, no considerando la importancia de éstos en toda la vida humana, por el contrario, muchos de los estudios comprenden, de manera implícita, los cuidados de forma instrumental, a punto tal que llega a ser inexistente una explicitación del significado de éstos.

Asimismo, llama nuestra atención que realizando un entrecruce respecto a las características de las personas cuidadas, los/as adultos/as mayores fueran altamente asociados a la enfermedad y las personas mayores, en que el tratamiento de esta 
población suele ser desde el enfoque biomédico, construyendo una vejez homogénea y asexuada, donde las diferencias abordadas entre mujeres y hombres son la esperanza de vida y a la afectación de enfermedades. En estos estudios, se puede apreciar que no existe una preocupación mayor sobre las relaciones de poder y las jerarquías de género en la ancianidad, invisibilizándose este período de la vida y a las personas que la viven, como actores sociales agenciados de sus propias vidas y bienestar, así como el de sus comunidades.

Por otro lado, las labores de cuidado no remunerado son realizadas principalmente por las mujeres de las familias, lo que confirma lo planteado por Elizabeth Jelin (2013), Karina Batthyány (2010), e Irma Arriagada y Rosalba Todaro (2012), respecto de la inexorable necesidad de incluir una perspectiva de género en la problemática de cuidados. Éstos suelen ejercerse por una obligación social y de género, que nos lleva a una dimensión normativa de los cuidados dada por quién debe cuidar, a quién debe cuidar, y por qué debe hacerlo. En este sentido, la presión social lleva a las mujeres a aceptar el cuidado como parte de sus atribuciones de género, implicando incluso el sacrificio de los intereses propios. En los artículos, los costos asociados de cuidar suelen traducirse en una identificación y descripción de efectos -generalmente, costos laborales, temporales y/o psiquiátricos y físicos-, no obstante, en menor medida es visibilizado el costo existencial de este cuidado sacrificial, que implica dar parte de la vida propia por el bienestar de otros/as de manera ilimitada. Lo anterior lo vinculamos a lo planteado por Simone de Beauvoir (1972), en tanto este cuidado conllevaría, sobre todo, una desresponsabilización de parte de quien cuida por su propia vida, intereses y actos. A esto añadimos una idea dialéctica entre la responsabilidad por las decisiones individuales, y las condiciones estructurales y sociales que enmarcan y condicionan dichas decisiones. Esto permite comprender el modo en que se configura y entiende el cuidado, así como quienes se responsabilizan por éste.

Por su parte, pese a la importancia que se le da en las perspectivas feministas a la interseccionalidad en los estudios de género y sexualidad (Wodak, 2015, entre otras), la revisión de la literatura dio cuenta de que esta perspectiva teórica no es considerada en los distintos abordajes, donde para el caso de la condición socio-económica, ésta solía entenderse desde un punto de vista descriptivo o como parte de las consideraciones finales, no ligándolo con una intersección de género. En este sentido, la pobreza solía comprenderse como un efecto del trabajo de cuidados no remunerado, no existiendo una problematización más profunda respecto a la propia categoría social, traduciéndose igualmente en la carencia de recursos económicos para externalizar el cuidado hacia otras mujeres en condiciones de mayor precariedad. Así, la respuesta mercantil no resultaba cuestionada en los artículos, pese a reproducir la consideración del cuidado como una responsabilidad familiar, individual (Castelló, 2009), y principalmente de mujeres.

Además, los estudios suelen identificar a un sujeto dependiente como parte del cuidado, pero ningún artículo empírico plantea ni se propone indagar el cuidado ejercido desde las 
mujeres, a hombres sanos, o "dependientes sociales" como refiere Sira del Río (2004). Estos últimos se ubican en una posición privilegiada con respecto a la responsabilidad por cuidar, pues no se espera socialmente que ejerzan el cuidado, donde la literatura pudo dar cuenta de que suelen recurrir a las redes formales de apoyo y/o mercantilizar el cuidado de manera mucho más frecuente, e incluso, en aquellos casos excepcionales que se responsabilizaban por el cuidado, estos hombres terminaban siendo elogiados. En este sentido, queremos relevar que las relaciones humanas deben ser entendidas desde la interdependencia, pese a su opacidad en relación a la exaltación de la autonomía individual como un valor sobre otras formas de vincularse y existir, de esta forma, pensamos que debe reflexionarse en torno a la reorganización social de los cuidados, desfamiliarizándolos, des-feminizándolos y volviéndolos colectivos.

Una alternativa para colectivizar los cuidados, es indagarlos, visibilizando las formas en que éstos se llevan a cabo, por ejemplo a través de organizaciones sociales y comunitarias. En este sentido, las prácticas comunitarias pueden ser una alternativa de respuesta a las crisis económicas globales, no obstante, pese a su importancia y potencialidad en la provisión de cuidados, los estudios que atienden a las mismas son incipientes. Nuestra revisión permitió dar cuenta de los escasos estudios existentes sobre la temática, cuyo abordaje era desde la niñez y las madres comunitarias, mas no en otras poblaciones, como las de personas mayores. Este olvido analítico podría pasar por los esquemas de conocimiento hegemónicos que definen y entienden de determinadas formas la vejez, como ya hemos mencionado anteriormente.

Es así como el ámbito comunitario se presenta como una alternativa para el estudio de cuidados como proceso colectivo, de reconocimiento, interdependencia y autogestión. La mirada del cuidado comunitario desde esta perspectiva, resalta una definición implícita relacionada con la interdependencia, la cual concuerda con nuestra propuesta feminista, en términos del desarrollo de la reciprocidad y la visión de la subjetividad construida a partir de lo intersubjetivo.

Por último, cabe señalar que parte de las limitaciones de nuestra revisión sistemática, corresponde a lo que Kay Dickersin (1994) denomina "sesgo de publicación científica", en tanto podemos suponer que gran parte de los artículos trabajados, responden al interés de la comunidad científica y de las propias revistas para publicar, aunque esto signifique omitir aspectos relevantes de la investigación. Lo anterior tiene por efecto, que dichos artículos terminan siendo concordantes con sus supuestos y/o hipótesis iniciales, omitiéndose tanto sus propias limitaciones, como aquellos aspectos que no concuerden con lo sostenido.

Por otra parte, en lo que concierne a las limitaciones propias del diseño de nuestra revisión, consideramos las fuentes utilizadas para la selección de artículos y las características de éstos. En este sentido, la exclusión de artículos teóricos pudo significar 
una debilidad, pues éstos pudiesen habernos brindado mayor diversidad de perspectivas al momento de abordar el problema indagado.

Por último, cabe mencionar la limitación geográfica establecida, ya que la revisión pudo haberse visto enriquecida con la inclusión de estudios más allá de la región Iberoamericana. Cabe señalar que, pese a que en esta región compartimos ciertos componentes culturales, no debemos perder de vista la heterogeneidad de experiencias que la constituyen, por lo que la vivencia de los cuidados dependerá del contexto y condiciones particulares de cada país.

\section{BIBLIOGRAFÍA}

Aguirre, Rosario (2008). El futuro del cuidado, en Arriagada, Irma (ed.): Futuro de las familias y desafíos para las políticas, Serie Seminarios y conferencias No. 52, p.2334. Santiago, Chile: CEPAL.

Aldana-González, Gabriela y García-Gómez, Liliana (2011). La experiencia de ser cuidadora de un anciano con enfermedad crónica, en Aquichan, Vol. XI, No. 2, p.158-172.

Andréu, Jaime (2000). Las técnicas de Análisis de Contenido: Una revisión actualizada [On Line]. Disponible en: http://public.centrodeestudiosandaluces.es/pdfs/S200103.pdf

Arriagada, Irma y Todaro, Rosalba (2012). El papel de las migrantes peruanas en la provisión de cuidados en Chile. Santo Domingo, República Dominicana: ONU Mujeres.

Barona-Vilar, Carmen; Más-Pons, Rosa; Fullana-Montoro, Ana; Giner-Monfort, Jordi; Grau-Muñoz, Arantxa y Bisbal-Sanz, Josep (2013). Perceptions and experiences of parenthood and maternal health care among Latin American women living in Spain: A qualitative study, en Midwifery, Vol. XXIX, No. 4, p.332-337.

Batthyány, Karina (noviembre, 2010). Envejecimiento, cuidados y género en América Latina. Trabajo presentado en Seminario internacional Experiencias internacionales y propuestas para consolidar la red nacional de cuidado de las personas adultas mayores en Costa Rica. Resumen disponible en: http://www.cepal.org/dds/noticias/paginas/3/41413/batthyany.pdf

Bedoya-Hernández, Mauricio (2013). Redes del cuidado: Ética del destino compartido en las madres comunitarias antioqueñas, en Revista Latinoamericana de Ciencias Sociales, Niñez y Juventud, Vol. XI, No. 2, p.741-753. 
Beltrán, Óscar (2005). Revisiones sistemáticas de la literatura, en Revista Colombiana de Gastroenterología, Vol. XX, No. 1, p.60-69.

Bolla, Bruna; Fulconi, Stéphany; Rigotti, Marja y Dupas, Giselle (2013). Cuidado da criança com anomalía congênita: a experiência da família, en Escola Anna Nery Revista de Enfermagem, Vol. XVII, No. 2, p.284-290.

Bustamante, Vania y McCallum, Cecilia (2011). Cuidado infantil na relação entre adultos e crianças na periferia de Salvador, en Psicologia \& Sociedade, Vol. XXIII, No. 3, p.506-515.

Carrion, Iraida y Nedjat-Haiem, Frances (2013). Caregiving for Older Latinos at End of Life: Perspectives From Paid and Family (Unpaid) Caregivers, en American Journal of Hospice and Palliative Medicine, Vol. XXX, No. 2, p.183-191.

Casado-Mejía, Rosa y Ruiz-Arias, Esperanza (2013). Estrategias de provisión de cuidados familiares a personas mayores dependientes, en Index Enfermería, Vol. XXII, No. 3, p.142-146.

Castelló, Laia (2009). La mercantilización y mundialización del trabajo reproductivo. El caso español, en Revista de Economía Crítica, No. 7, p.74-94.

Comas D’Argemir, Dolors (2014). Los cuidados y sus máscaras: retos para la antropología feminista, en Mora, Vol. XX, No. 1, p.167-182.

Da Silva, Mara; Luz, Geisa; Cezar-Vaz, Marta y Silva, Priscila (2012). Trabalho familiar: distribuição desejada do trabalho doméstico e cuidados dos filhos entre cônjuges, en Revista Gaúcha de Enfermagem, Vol. XXXIII, No. 1, p.124-131.

De Beauvoir, Simone (1972). The second sex. Harmondsworth, UK: Penguin Books.

De São José, José (2012). A divisão dos cuidados sociais prestados a pessoas idosas: Complexidades, desigualdades e preferěncias, en Sociologia, Problemas e Praticas, Vol. LXIX, p.63-85.

De Sousa, Lívia; Parente, Ana y Gomes, Edilma (2009). Paciente com Acidente Vascular Encefálico e a rede de apoio familiar, en Revista Brasileira de Enfermagem, Vol. LXII, No. 2, 271-277.

De Souza, Nilba; Pinheiro-Fernandes, Ana; Clara-Costa, Íris; Cruz-Enders, Bertha; Leite de Carvalho, Jovanka y Costa da Silva, María (2010). Domestic maternal experience with preterm newborn children, en Revista de Salud Pública, Vol. XII, No. 3, p.356367. 
Del Río, Sira (2004). La crisis de los cuidados: precariedad a flor de piel, en Rebelión Economía, No. 1 , [On Line]. Disponible en: https://www.rebelion.org/hemeroteca/economia/040308sira.htm

Del Río-Lozano, María; García-Calvente, María; Marcos-Marcos, Jorge; Entrena-Durán, Francisco y Maroto-Navarro, Gracia (2013). Gender identity in informal care: Impact on health in Spanish caregivers, en Qualitative Health Research, Vol. XXIII, No. 11, p.1506-1520.

Dickersin, Kay (1994). Sobre la existencia y los factores de riesgo del sesgo de publicación, en Bol Oficina Sanit Panam, Vol. CXVI, No. 5, p.435-446.

Dos Anjos, Karla; Boery, Rita y Pereira, Rafael (2014). Quality of life of relative caregivers of elderly dependents at home, en Texto e Contexto Enfermagem, Vol. XXIII, No. 3, p.600-608.

Dos Santos, Ariene y lost, Sofía (2010). Perfil dos cuidadores de idosos com alterações cognitivas em diferentes contextos de vulnerabilidade social, en Revista Gaúcha de Enfermagem, Vol. I, No. 1, p.115-122.

Dos Santos, Ariene y lost, Sofía (2012). Funcionalidade familiar de idosos com alterações cognitivas: a percepção do cuidador, en Revista da Escola de Enfermagem da USP, Vol. XL, No. 5, p.1141-1147.

Ezquerra, Sandra (2011). Crisis de los cuidados y crisis sistémica: la reproducción como pilar de la economía llamada real, en Investigaciones Feministas, Vol. II, p.175-194.

Fernandes de Oliveira, Eteniger y Santana de Brito, Rosineide (2009). Ações de cuidado desempenhadas pelo pai no puerpério, en Escola Anna Nery Revista de Enfermagem, Vol. XIII, No. 3, p.595-601.

Flores, Roberta y Tena, Olivia (2014). Maternalismo y discursos feministas latinoamericanos sobre el trabajo de cuidados: un tejido en tensión, en Íconos. Revista de Ciencias Sociales, No. 50, p.27-42.

Fratezi, Flavia y Gutierrez, Beatriz (2011). Cuidador familiar do idoso em cuidados paliativos: O processo de morrer no domicílio, en Ciencia e Saude Coletiva, Vol. XVI, No. 7, p.3241-3248.

Gálvez, María; Ríos, Francisca y García, Ana (2013). Crecer cuidando. El rol cuidador en las hijas de madres dependientes, en Index de enfermería, Vol. XXII, No. 4, p.237-241. 
Guerini, Isabelle; Cordeiro, Priscilla; Zirke, Samantha y Ribeiro, Edilza (2012). Percepção de familiares sobre estressores de correntes das demandas de cuidado de criança e adolescente dependentes de tecnologias, en Texto e Contexto Enfermagem, Vol. XXI, No. 2, p.348-355.

Henn, Camila y Piccinini, César (2010). A Experiência da Paternidade o Envolvimento Paterno no Contexto da Síndrome de Down, en Psicologia: Teoria e Pesquisa, Vol. XXVI, No. 4, p.623-631.

Instituto Nacional de Estadísticas (2016a). Género y Empleo. Enfoque Estadístico. Disponible en: http://www.ine.cl/genero/files/estadisticas/pdf/documentos/enfoque_genero_20 16.pdf

Instituto Nacional de Estadísticas (2016b). Encuesta Nacional Sobre Uso del Tiempo. Documento de Principales Resultados ENUT 2015. Disponible en: http://www.ine.cl/enut/files/principales_resultados/documento_resultados_ENU T.pdf

Izquierdo, María Jesús (2003). Del sexismo y la mercantilización del cuidado a su socialización: Hacia una política democrática del cuidado. En Congreso Internacional SARE, Cuidar cuesta: costes y beneficios del cuidado, p.1-30. Barcelona: Emakunde/Instituto Vasco de la mujer.

Jelin, Elizabeth (2013). Políticas de cuidado. Debates conceptuales en torno al cuidado. La dimensión de género en las políticas de cuidado. Trabajo presentado en Diálogos sobre Políticas de Cuidado en la Argentina. Resumen disponible en: http://www.revistahumanum.org/revista/wpcontent/uploads/2013/09/Dialogos_marzoyabril1.pdf

Krmpotic, Claudia y De leso, Lia (2010). Los cuidados familiares. Aspectos de la reproducción social a la luz de la desigualdad de género, en Revista Katálysis, Vol. XIII, p.95-101.

Larrañaga, Isabel; Valderrama, María; Martín, Unai; Begiristain, José; Bacigalupe, Amaia y Arregi, Begoña (2009). Mujeres y hombres ante el cuidado informal: diferencias en los significados y las estrategias, en Revista Facultad Nacional de Salud Pública, Vol. XXVII, No. 1, p.50-55.

Letablier, Marie-Thérèse (2007). El trabajo de «cuidados» y su conceptualización en Europa, en Prieto, Carlos (ed.): Trabajo, Género y tiempo social, p. 64-84. Madrid, España: Editorial Complutense. 
Magaña, Irene; Calquín, Claudia; Silva, Sebastián y García, Makarena (2011). Diversidad Familiar, Relaciones de Género y Producción de Cuidados en Salud en el Modelo de Salud Familiar: Análisis de Caso en un CESFAM de la Región Metropolitana, Chile, en Terapia Psicológica, Vol. XXIX, No. 1, p.33-42.

Martínez, Juliana y Voorend, Koen (2011). Sistemas de patriarcado y regímenes de bienestar en América Latina: ¿Una cosa lleva a la otra? Documento de Trabajo No. 37. Madrid, España: Fundación Carolina - CeALCI.

Moreno-Zavaleta, María y Granada-Echeverri, Patricia (2014). Interacciones vinculares en el sistema de cuidado infantil, en Revista Latinoamericana de Ciencias Sociales, Niñez y Juventud, Vol. XII, No. 1, p.121-139.

Razavi, Shahra (2007). The Political and Social Economy of Care in a Development Context: Conceptual Issues, Research Questions and Policy Options. Génova, Italia: UNRISD.

Rodríguez, María; Peña, José-Vicente y Torío, Susana (2014). La experiencia de la paternidad y la maternidad: análisis del discurso de las creencias sobre la crianza y el cuidado infantil, en Infancia y Aprendizaje: Journal for the Study of Education and Development, Vol. XXXII, No. 1, p.81-95.

Sanches, Leila (2012). Working Mothers and their Multivoiced Self, en Revista Colombiana de Psicología, Vol. XXI, No. 2, p.315-324.

Sen, Amartya (2000). Desarrollo y Libertad. Buenos Aires: Editorial Planeta.

Silva, Silvana; Budó, Maria y Da Silva, Marciele (2013). Concepções e práticas de cuidado na visão de homens, en Texto e Contexto Enfermagem, Vol. XXII, No. 2, p.389-396.

Sousa, Joana; Silva, Abel y Marques, Paulo (2011). Estratégias do membro da família prestador de cuidados face às manifestações de confusão do seu familiar, en Revista de Enfermagem Referência, Vol. III, No. 4, p.59-67

Stackfleth, Renata; Aleixo, Mariana; Silva, Mariana; Vendruscolo, Thais; Fabrício-Whebe, Suzele; Marques, Sueli y Rodrigues, Rosalina (2012). Sobrecarga de trabalho em cuidadores de idosos fragilizados que vivem no domicílio, en ACTA Paulista de Enfermagem, Vol. XXV, No. 5, p.768-74.

Tarrés, María Luisa (2004). Observar, escuchar y comprender. Sobre la tradición cualitativa en la investigación social. México: FLACSO. 
Tobío, Constanza; Agulló, María Silveria; Gómez, María Victoria y Martín, María Teresa (2010). El cuidado de las personas. Un reto para el siglo XXI. Colección Estudios Sociales Núm. 28. Barcelona, España: Fundación "la Caixa".

Torns, Teresa (2008). El trabajo y el cuidado: cuestiones teórico-metodológicas desde la perspectiva de género, en EMPIRIA. Revista de Metodología de Ciencias Sociales, No.15, p.53-73.

Tronto, Joan (1993). Moral boundaries: A political argument for an ethics of care. New York: Routledge

Truzzi, Annibal; Valente, Letice; Ulstein, Ingun; Engelhardt, Eliasz; Laks, Jerson y Engedal, Knut (2012). Burnout in familial caregivers of patients with dementia, en Revista Brasileira de Psiquiatria, Vol. XXXIV, No. 4, p.405-412.

Urrútia, Gerard y Bonfill, Xavier (2010). Declaración PRISMA: una propuesta para mejorar la publicación de revisiones sistemáticas y metaanálisis, en Medicina Clínica, Vol. CXXXV, No. 11, p.507-511.

Vieira, Lizyana; Rodrigues, Joseane; Correia, Carme y Okuda, Keila. (2012). Cuidar de um familiar idoso dependente no domicílio: reflexões para os profissionais da saúde, en Revista Brasileira Geriatria e Gerontologia, Vol. XV, No. 2, p.255-263.

Villarejo, Luis; Zamora, María Antonia y Casado, Gaspar (2012). Sobrecarga y dolor percibido en cuidadoras de ancianos dependientes, En Enfermería Global, Vol. XI, No. 27, p.159-165.

Weisbrot, Mariela y Giraudo, Nanci (2012). Conceptos y percepciones de las abuelas sobre el cuidado de sus nietos. Estudio cualitativo en una población del Hospital Italiano de Buenos Aires, en Archivos Argentinos Pediatría, Vol. CX, No. 2, p.126131.

Wodak, Ruth (2015). Gender and Language: Cultural Concerns, en James D. Wright (ed.): International Encyclopedia of Social \& Behavioral Sciences, p.698-703. Oxford, UK: Elsevier.

Wottrich, Shana y Arpini, Dorian (2014). Cuidados necessários à infância: um estudo com mães coletadoras de material reciclável, en Temas em Psicologia, Vol. XXII, No. 2, p.471-482. 